\title{
A PROBABILISTIC REMARK ON ALGEBRAIC PROGRAM TESTING
}

\author{
Richard A. DEMILLO \\ School of Information and Computer Science, Georgia Institute of Technology, Atlanta, GA 30332, USA
}

\author{
Richard J. LIPTON \\ Computer Science Department, Yale University, New Haven, CT 06520, USA
}

Received 8 August 1977; revised version received 27 March 1978

Sof tware reliability, program testing

Until very recently, research in software reliability has divided quite neatly into two - usually warring camps: methodologies with a mathematical basis and methodologies without such a basis. In the former. view, "reliability" is identified with "correctness" and the principle tocl has been formal and informal verification [1]. In the latter view, "reliability" is taken to mean the ability to meet overall functional goals to within some predefined limits $[2,3]$. We have argued in [4] that the latter view holds a great deal of promise for further development at both the practical and analytical levels. Howden [5] proposes a first step in this direction by describing a method for "testing" a certain restricted class of programs whose behavior can - in a sense Howden makes precise :be algebraicized. In this way, "testing" a program is reduced to an equivalence test, the major components of which beiome

(i) a combinatorial identification of "equivalent" structures;

(ii) an algebraic test

$$
f_{1} \equiv f_{2},
$$

where $f_{i}, i=1,2$ is a multivariable polynornial (multinomial) of degree specified by the program being considered.

In arriving at a method for exact solution of (ii), Howden derives an algorithm, that requires evaluation of multinomials $f\left(x_{1}, \ldots, x_{m}\right)$ of maximal degree $c^{\prime}$ at $O\left[(d+1)^{m}\right]$ proints. For large values of $m$ (a typical case for realistic examples), this method becomes prohibitively expensive.

Since, however, a test for reliability rather than a certification of correctness is dusired, a natural question is whether or not Howden's method can be improved by settling for less than an exact solution to (ii).

We are inspired by Rabin [6] and, less directly, by the many successes of Erdös and Spencer [7] to attempt a probabilistic solution to (ii). Using thicse methods, we show that (ii) an be tested with probability of error $\epsilon$ * with only $\mathcal{U}(g(\epsilon))$ evaluations of multinomials, where $g$ is a slowly growing function of only $\epsilon$. In particular, 30 or sc evaluations shorid give sufficiently small probability of error for mist practicai situations. The remainder of this note is devoted to proving this result.

Let us denote by $P_{\neq 0}(m, d)$ the class of multinumials

$f\left(x_{1}, \ldots, x_{m}\right) \not \equiv 0$

(over some arbitrary but íxed integral domain) whose degree does not exceed $d>0$. We define

$P(m, d, r)=\min \operatorname{Prob}\left\{1 \leqslant x_{1} \leqslant r, f\left(x_{1}, \ldots, x_{m}\right) \neq 0\right\}$

$f \in P_{\neq 0}(m, d)$.

\footnotetext{
* See Rabin's account of algorithms inat may err with fixed probability [6].
} 
We think of $P(m, d, r)$ as the minimal relative frequency with which witnesses to the non-nullity of a multinomial of the appropriate ?ind can occur in the chosen interval. We will derive a lower bound $p$ for $P(m, d, r)$. Then $(1-p)$ is an upper bound on the error in selecting a random point from the $m$-cube. We then iterate the procedure by $t$ independent random selections to obtain a small probability of error $(1-p)^{t}$. Notice, in particular, that since a polynomial of degree $d$ has at most $d$ roots (ignoring multiplicity), the largest probability of finding a root must be at least the probability of finding a root by randomly sampling in the interval $1 \leqslant x_{1} \leqslant r$; thus

$P(1, d, r) \geqslant 1-d / r$.

Now, consider some

$f\left(x_{1}, \ldots, x_{m}, y\right) \neq 0$

of degree at most $d$. But there are then multinomials

$\left\{g_{i}\right\}_{i<d}$, not all $\neq 0$, such that

$f\left(x_{1}, \ldots, x_{m}, y\right)=\sum_{i=0}^{d} g_{i}\left(x_{1}, \ldots, x_{m}\right) y^{i}$.

Let us suppose that $g_{k} \in P_{\neq 0}(m, d)$. Thus

$\operatorname{Prob}\left\{1 \leqslant x_{i} \leqslant r, f\left(x_{1}, \ldots, x_{n}, y\right) \neq 0\right\}$

$\geqslant \operatorname{Prob}\left\{g_{k}\left(x_{1}, \ldots, x_{m}\right) \neq 0\right.$ and $y$ is not a root $\}$

$\geqslant P(m, d, r)(1-d / r)$.
Continuing inductively, we obtain a lower bound in $P(m, d, r)$ as follows:

$P(m, d, r) \geqslant(1-d / r)^{m}$.

But

$$
\begin{aligned}
& \lim _{m \rightarrow \infty}(1-d / r)^{m}=\lim _{m \rightarrow \infty}\left[1+\frac{1}{m}\left(\frac{-d m}{r}\right)\right]^{m} \\
& =\exp (-d m / r)
\end{aligned}
$$

Combining (1) and (2), we have for large $m, r=d m$, $P(m, d, d m) \geqslant \mathrm{e}^{-1}$.

Thus, with $t$ evaluations of $f$ for independent choices of points from the $m$-cube with sides $r=d m$, the probability of missing a witness to the non-nullity of $f\left(x_{1}, \ldots, x_{m}\right)$ is at most

$\left(1-\mathrm{e}^{-1}\right)^{t}$.

Table 1 shows the probable error in testing $f \equiv 0$ by $t$ evaluations of $f$ at randomly chosen points for some typical values of $d, m, r, t$. Notice that for $d m=r, t=30$, this is already $<10^{-5}$.

\section{References}

[1] Z. Manna, Mathematical Theory of Computation (McGraw-Hill, New York, 1974).

Table 1

Probable error in testing $f\left(x_{1}, \ldots, x_{m}\right) \equiv 0$ (degree $<d$ ) by $t$ random evaluations in $\{1, \ldots, r\}$

\begin{tabular}{lllllll}
\hline$[1-P(m, d, r)]^{t}$ & & & & \\
\hline$d m$ & $r$ & $t+10$ & $t=20$ & $r=30$ & $t=50$ & $t=100$ \\
\hline 10 & 10 & $10 \times 10^{-3}$ & $106 \times 10^{-6}$ & $1 \times 10^{-6}$ & $109 \times 10^{-12}$ & $12 \times 10^{-21}$ \\
20 & 10 & $233 \times 10^{-3}$ & $54 \times 10^{-3}$ & $13 \times 10^{-3}$ & $695 \times 10^{-6}$ & $483 \times 10^{-9}$ \\
50 & 10 & $935 \times 10^{-3}$ & $873 \times 10^{-3}$ & $816 \times 10^{-3}$ & $713 \times 10^{-3}$ & $509 \times 10^{-3}$ \\
$10^{2}$ & 10 & $\sim 1$ & $\sim 1$ & $\sim 1$ & $\sim 1$ & $\sim 1$ \\
10 & $10^{2}$ & $61 \times 10^{-12}$ & $<10^{-20}$ & $<10^{-20}$ & $<10^{-20}$ & $\sim 0$ \\
20 & $10^{2}$ & $38 \times 10^{-9}$ & $1 \times 10^{-15}$ & $<10^{-20}$ & $<10^{-20}$ & $\sim 0$ \\
50 & $10^{2}$ & $88 \times 10^{-6}$ & $8 \times 10^{-9}$ & $704 \times 10^{-15}$ & $<10^{-20}$ & $<10^{-20}$ \\
$10^{3}$ & $10^{2}$ & $\sim 1$ & $\sim 1$ & $\sim 1$ & $\sim 1$ & $\sim 1$ \\
10 & $10^{3}$ & $<10^{-20}$ & $<10^{-20}$ & $<10^{-20}$ & $\sim 0$ & $\sim 0$ \\
20 & $10^{3}$ & $9 \times 10^{-18}$ & $<10^{-20}$ & $<10^{-20}$ & $\sim 0$ & $\sim 0$ \\
50 & $10^{3}$ & $76 \times 10^{-15}$ & $<10^{-20}$ & $<10^{-20}$ & $\sim 0$ & $\sim 0$ \\
\hline
\end{tabular}


12] J.R. Brown, M. Lipow, Testing for software reliabikity, Intern. Conf, in Reliable Software, SIGPLAN Notices, 10, b, (June 1975) 518-527.

[3] A.I. Llewelyn, R.F. Wilkins, The testing of computer software, 1969 Conf. on Software Engineering, 189-199.

[4] R.A. DeMillo, R.J. Lipton, A.J. Perlis, Social processes and proofs of theorems and programs, Fourth ACM Symposium in Principles of Programming Languages (to appear in CACM).
[5] W.F. Howden, Algebraic program testing, Computer Science Teshnical Report No. 14 (November 1976) UC-San Diego, La Jolla, CA.

[6] M.O. Rabin, Probabilistic algorithms, in: J. Traub, ed., Algorithms and Complexity (Academic Press, New York, 1976) $21-40$.

[7] P. Erdös, J. Spencer, Probabilistic Methois in Combinatorics (Academic Press, New Yorik, 1974). 